TITLE:

\title{
New evaluation of reconstructed spatial distribution function from radial distribution functions
}

$\operatorname{AUTHOR}(\mathrm{S}):$

Yokogawa, D; Sato, H; Sakaki, S

\section{CITATION:}

Yokogawa, D ... [et al]. New evaluation of reconstructed spatial distribution function from radial distribution functions. JOURNAL OF CHEMICAL PHYSICS 2006, 125(11): 114102.

\section{ISSUE DATE:}

2006-09-21

URL:

http://hdl.handle.net/2433/39771

\section{RIGHT:}

Copyright 2006 American Institute of Physics. This article may be downloaded for personal use only. Any other use requires prior permission of the author and the American Institute of Physics. 


\title{
New evaluation of reconstructed spatial distribution function from radial distribution functions
}

\author{
Daisuke Yokogawa, Hirofumi Sato, ${ }^{\text {a) }}$ and Shigeyoshi Sakaki \\ Department of Molecular Engineering, Graduate School of Engineering, Kyoto University, Nishikyo-ku, \\ Kyoto 615-8510, Japan
}

(Received 12 April 2006; accepted 3 August 2006; published online 18 September 2006)

\begin{abstract}
Although three dimensional (3D) solvation structure is much more informative than one dimensional structure, its evaluation is difficult experimentally and theoretically. In our previous Communication [Yokogawa et al., J. Chem. Phys. 123, 211102 (2005)], we proposed a new method to present reconstructed spatial distribution function (RC-SDF) from a set of radial distribution functions (RDFs). In this article, we successfully extended the method more accurately with new basis sets. This new method was applied to two liquid solvation structures, methanol and dimethyl sulfoxide, as examples. Their RC-SDFs evaluated here clearly show that the former solvation structure is well defined while the latter one is broad, which agrees well with the SDFs calculated directly from molecular dynamics simulations. These results indicate that the method can reproduce well these 3D solvation structures in reasonable computational cost. (C) 2006 American Institute of Physics. [DOI: 10.1063/1.2345199]
\end{abstract}

\section{INTRODUCTION}

Local solvation structure such as hydrogen bonding has great influence on the stabilities of compounds and their electronic structures. ${ }^{1}$ To investigate such solvation structures, three dimensional (3D) solvation structure is very useful.

In this regard, 3D solvation structure has been studied by theoretical methods. For instance, 3D structures of pure solvent $^{2,3}$ and binary solvent ${ }^{4}$ were calculated directly with molecular dynamics (MD) method. Also, the integral equation theory (IET) ${ }^{5}$ such as molecular Ornstein-Zernike ${ }^{6,7}$ (MOZ) and 3D reference interaction site model (3D-RISM),${ }^{8-10}$ were proposed to provide the $3 \mathrm{D}$ structures.

There is another approach to obtain 3D solvation structure. ${ }^{11-15}$ The strategy is to reconstruct 3D solvation structure from a set of one dimensional (1D) solvation structures such as radial distribution functions (RDFs). Because the 1D solvation structure that is averaged over molecular orientations can be easily presented experimentally and theoretically, the method to reconstruct $3 \mathrm{D}$ solvation structure from 1D data is very powerful to analyze solvation structure. Actually, Soper et al. expanded the angular pair-correlation functions with spherical harmonics and determined the coefficients with the maximum-entropy method. ${ }^{11-13}$ Sato and Hirata proposed most plausible solvation structure (MPSS) method, ${ }^{14}$ in which they determined the MPSS from a set of RDFs and also represented thermal fluctuation around MPSS with Gaussian functions. Recently, we have proposed a new method to obtain reconstructed spatial distribution function (RC-SDF) from RDFs. ${ }^{15}$ In the method, we expanded spatial distribution function (SDF) using real solid harmonics and Gaussian functions on each solute site. This method was successfully applied to water, in which clear picture of the 3D

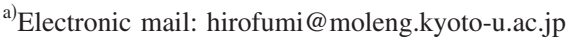

solvation structure was presented. In the previous work, a few Gaussian functions were employed to represent the radial part so as to reproduce the reference RDFs of water. This strategy is very useful when limited number of Gaussian functions are employed; for example, the RDF of water was reproduced well, as reported. However, we need to increase the number of Gaussian functions systematically in order to improve the quality of RC-SDF when the solvation structure is not simple. In our previous method, ${ }^{15}$ the computational time becomes very long as a number of Gaussian functions increase.

To overcome this weakness, we refined our previous method to adopt a new type of basis sets. We applied the new method to analyze the liquid structures of methanol and dimethyl sulfoxide (DMSO). We selected these solvents as examples, because it is said that the former solvation structure is well defined and the latter one is broad. The results presented here are discussed in comparison with SDF directly calculated by the MD method.

\section{METHOD}

SDF of solvent site $s, n_{s}(\mathbf{r})$, can be expanded at each solute site $\eta$ by using the real solid harmonics $\left\{Y_{n m}(\theta, \phi)\right\}$ as follows:

$$
\left.n_{s}(\mathbf{r})\right|_{\eta}=\sum_{n=0}^{\infty} \sum_{m=-n}^{n} N_{n m}^{\eta, s}\left(\left|\mathbf{r}-\mathbf{Q}_{\eta}\right|\right) Y_{n m}(\theta, \phi)
$$

where $N_{n m}^{\eta, s}\left(\left|\mathbf{r}-\mathbf{Q}_{\eta}\right|\right)$ is the radial function around the $\eta$ site. This function can be expanded with one dimensional functions $\left\{f_{i}^{\eta, s}\right\}$ and $f^{\eta, s}$, 


$$
\begin{aligned}
N_{n m}^{\eta, s}\left(\left|\mathbf{r}-\mathbf{Q}_{\eta}\right|\right) \simeq & \sum_{i}^{N} C_{i, n, m}^{\eta, s} f_{i}^{\eta, s}\left(\left|\mathbf{r}-\mathbf{Q}_{\eta}\right|\right)\left(1-\delta_{n 0} \delta_{m 0}\right) \\
& +\sqrt{4 \pi} f^{\eta, s}\left(\left|\mathbf{r}-\mathbf{Q}_{\eta}\right|\right) \delta_{n 0} \delta_{m 0},
\end{aligned}
$$

where $\left\{C_{i, n, m}^{\eta, s}\right\}$ are the coefficients to be determined, $\left\{f_{i}^{\eta, s}\right\}$ are spatially well localized functions which reproduce anisotropic solvent structure $(n, m \neq 0)$, and $f^{\eta, s}$ is the function which represents isotropic one $(n, m=0)$. Insertion of Eq. (2) into Eq. (1) gives the following equation:

$$
\begin{aligned}
\left.n_{s}(\mathbf{r})\right|_{\eta}= & \sum_{i}^{N} f_{i}^{\eta, s}\left(\left|\mathbf{r}-\mathbf{Q}_{\eta}\right|\right) \sum_{n=1}^{\infty} \sum_{m=-n}^{n} C_{i, n, m}^{\eta, s} Y_{n m}(\theta, \phi) \\
& +f^{\eta, s}\left(\left|\mathbf{r}-\mathbf{Q}_{\eta}\right|\right) .
\end{aligned}
$$

These coefficients $\left\{C_{i, n, m}^{\eta, s}\right\}$ are determined under the following conditions. By integrating angular part of $\left.n_{s}(\mathbf{r})\right|_{\eta}$ around the $\eta$ site, the RDF must be reproduced (condition I). The sets of $\left\{\left.n_{s}(\mathbf{r})\right|_{\eta}\right\}$ at each solute site must be consistent with each other in 3D space (condition II). $\left.n_{s}(\mathbf{r})\right|_{\eta}$ must be positive in 3D space (condition III).

The integration of $\left.n_{s}(\mathbf{r})\right|_{\eta}$ around the $\eta$ site leads to the following equation:

$$
\begin{aligned}
\left.\frac{1}{4 \pi} \int_{0}^{2 \pi} d \phi \int_{0}^{\pi} \sin \theta d \theta n_{s}(\mathbf{r})\right|_{\eta} \\
=\sum_{i}^{N} f_{i}^{\eta, s}\left(\left|\mathbf{r}-\mathbf{Q}_{\eta}\right|\right) \sum_{n=1}^{\infty} \sum_{m=-n}^{m=n} \frac{C_{i, n, m}^{\eta, s}}{4 \pi} \int_{0}^{2 \pi} d \phi \\
\quad \times \int_{0}^{\pi} \sin \theta d \theta Y_{n, m}(\theta, \phi)+f^{\eta, s}\left(\left|\mathbf{r}-\mathbf{Q}_{\eta}\right|\right) \\
=f^{\eta, s}\left(\left|\mathbf{r}-\mathbf{Q}_{\eta}\right|\right),
\end{aligned}
$$

where we used the orthogonality condition of real solid harmonics. If $f^{\eta, s}$ is the RDF between the solvent $s$ site and the solute $\eta$ site $\left(f^{\eta, s}\left(\left|\mathbf{r}-\mathbf{Q}_{\eta}\right|\right) \equiv g^{\eta, s}\left(\left|\mathbf{r}-\mathbf{Q}_{\eta}\right|\right)\right)$ condition I is satisfied.

To satisfy condition II, we determined the coefficients so as to minimize the following $\Gamma^{\prime}$ value: ${ }^{15}$

$$
\Gamma^{\prime}=\frac{1}{2} \sum_{k}^{N_{p}} \sum_{\eta \neq \gamma}^{N_{a}}\left(\left.n_{s}\left(\mathbf{r}_{k}\right)\right|_{\eta}-\left.n_{s}\left(\mathbf{r}_{k}\right)\right|_{\gamma}\right)^{2}
$$

where $N_{p}$ is the number of grid point around solute site and $N_{a}$ is the number of solute sites.

To keep $n_{s}(\mathbf{r})$ positive, we adopted the following strategy. In the calculation of real solid harmonics, we divided a sphere into small regions $\Delta_{k}(k=1 \sim M)$ using a reduced grid system $^{16}$ (Fig. 1) and approximated the real solid harmonics by

$$
Y_{n, m}(\theta, \phi) \simeq \sum_{k} U_{k, n, m} \delta_{k}(\theta, \phi),
$$

where the function $\delta_{k}(\theta, \phi)$ is defined as

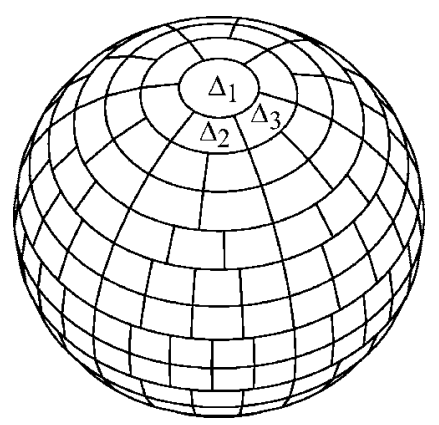

FIG. 1. Reduced grid sphere in the case of $M=254$. Note that we used $M$ $=12302$ in the present work.

$$
\delta_{k}(\theta, \phi)= \begin{cases}1 & \text { when }(\theta, \phi) \in \Delta_{k} \\ 0 & \text { when }(\theta, \phi) \notin \Delta_{k} .\end{cases}
$$

$\left\{U_{k, n, m}\right\}$ are the matrix elements which can be calculated using real solid harmonics. In this work, we used the reduced grid system $(M=12302)$. Insertion of Eq. (6) into Eq. (1) leads to the following equation for a given $\mathbf{r}_{j}=\left(r_{j}, \theta_{j}, \phi_{j}\right)$ :

$$
\left.n_{s}\left(\mathbf{r}_{j}\right)\right|_{\eta} \simeq \sum_{k}^{M} S_{j, k}^{\eta, s} \delta_{k}\left(\theta_{j}, \phi_{j}\right),
$$

where $S_{j, k}^{\eta, s}$ is defined by

$$
\begin{aligned}
S_{j, k}^{\eta, s}= & \sum_{i}^{N} f_{i}^{\eta, s}\left(\left|\mathbf{r}_{j}-\mathbf{Q}_{\eta}\right|\right) \sum_{n=1}^{\infty} \sum_{m=-n}^{n} C_{i, n, m}^{\eta, s} U_{k, n, m} \\
& +\sqrt{4 \pi} U_{k, 0,0} f^{\eta, s}\left(\left|\mathbf{r}_{j}-\mathbf{Q}_{\eta}\right|\right) .
\end{aligned}
$$

To satisfy condition III, we employed $\Gamma$ defined by Eq. (10) instead of $\Gamma^{\prime}$ [Eq. (5)],

$$
\Gamma=\frac{1}{2} \sum_{k}^{N_{p}} \sum_{\eta \neq \gamma}^{N_{a}}\left(\left.n_{s}\left(\mathbf{r}_{k}\right)\right|_{\eta}-\left.n_{s}\left(\mathbf{r}_{k}\right)\right|_{\gamma}\right)^{2}+2 \sum_{j, k, \eta} \phi\left(S_{j, k}^{\eta, s}\right),
$$

where $\phi$ is the penalty function. The value of the penalty function is 0 when $S_{j, k}^{\eta, s}$ is positive but monotonically increases as the value of $\left|S_{j, k}^{\eta, s}\right|$ increases. If $S_{j, k}^{\eta, s}$ is positive all over the space, Eq. (10) reduces to Eq. (5). We will discuss the penalty function in detail below. By minimizing $\Gamma$, we reach Eq. (11), with which we can obtain the coefficients $\left\{C_{i, n, m}^{\eta, s}\right\}$,

$$
\begin{aligned}
& \sum_{\beta, j, n \prime, m^{\prime}} A_{(s) \beta, j, n^{\prime}, m^{\prime}}^{\alpha, i, n, m} C_{j, n^{\prime}, m^{\prime}}^{\beta, s} \\
& \quad=-B_{(s)}^{\alpha, i, n, m}-\sum_{j, k} \frac{\partial \phi\left(S_{j, k}^{\alpha, s}\right)}{\partial S_{j, k}^{\alpha, s}} f_{i}^{\alpha, s}\left(\left|\mathbf{r}_{j}-\mathbf{Q}_{\alpha}\right|\right) U_{k, n, m},
\end{aligned}
$$

where $A_{(s) \beta, j, n^{\prime}, m^{\prime}}^{\alpha, i, m}$ and $B_{(s)}^{\alpha, i, n, m}$ are represented, respectively, by ${ }^{17}$

$$
\begin{aligned}
A_{(s) \beta, j, n^{\prime}, m^{\prime}}^{\alpha, i n, m}= & \left(N_{a} \delta_{\alpha, \beta}-1\right) \sum_{k}^{N_{p}} f_{i}^{\alpha, s}\left(\left|\mathbf{r}_{k}-\mathbf{Q}_{\alpha}\right|\right) f_{j}^{\beta, s}\left(\left|\mathbf{r}_{k}-\mathbf{Q}_{\beta}\right|\right) \\
& \times Y_{n m}\left(\theta_{k}, \phi_{k}\right) Y_{n^{\prime} m^{\prime}}\left(\theta_{k}, \phi_{k}\right),
\end{aligned}
$$




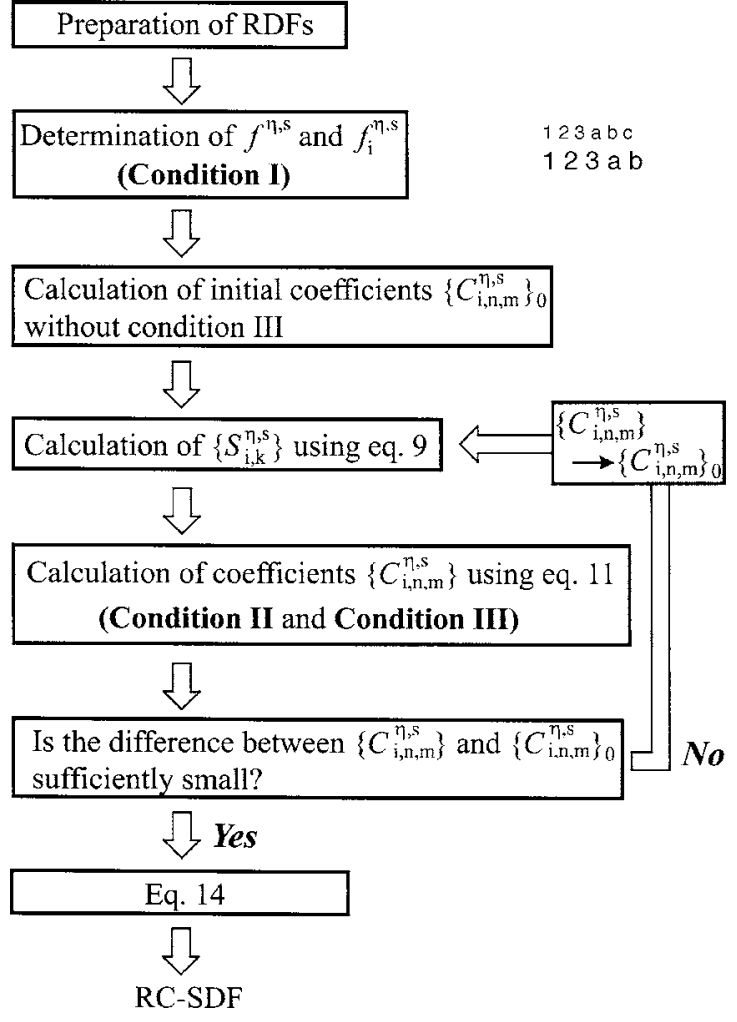

SCHEME 1.

$$
\begin{aligned}
B_{(s)}^{\alpha, i, n, m}= & \sum_{k=1}^{N_{p}} f_{i}^{\alpha, s}\left(\left|\mathbf{r}_{k}-\mathbf{Q}_{\alpha}\right|\right) Y_{n m}\left(\theta_{k}, \phi_{k}\right) \sum_{\gamma}^{N_{a}}\left(N_{a} \delta_{\alpha, \gamma}-1\right) \\
& \times f^{\gamma, s}\left(\left|\mathbf{r}_{k}-\mathbf{Q}_{\gamma}\right|\right)
\end{aligned}
$$

In our previous work, condition III was not considered because a set of $\left\{\left.n_{s}(\mathbf{r})\right|_{\eta}\right\}$ was positive almost all over the space, and the equation to be solved was linear. Condition III becomes important as the number of the functions $\left\{f_{i}^{\eta, s}\right\}$ increases. Because of condition III, Eq. (11) is not a linear equation here. Thus, we solved it iteratively. The initial guess is calculated by solving a linear equation [Eq. 6 in Ref. 15] because the results provided correct shape of SDF as shown in our previous work.

The sets of $\left\{\left.n_{s}(\mathbf{r})\right|_{\eta}\right\}$ are different from each other at $\mathbf{r}$. To obtain final RC-SDF $n_{s}(\mathbf{r})$ from these values, we used the following equation with proper weight, $w_{\alpha}(\mathbf{r})$ :

TABLE I. Intermolecular potential parameters. Molecular geometry: (methanol) $r_{\mathrm{OH}}=0.945 \AA, r_{\mathrm{CO}}=1.430 \AA$, and $\angle \mathrm{COH}=108.5^{\circ}$; (DMSO) [crystallographic data (Ref. 30)] $r_{\mathrm{OS}}=1.53 \AA, \quad r_{\mathrm{SC}}=1.80 \AA, \quad \angle \mathrm{OSC}$ $=106.75^{\circ}$, and $\angle \mathrm{CSC}=97.40^{\circ}$.

\begin{tabular}{llclr}
\hline \hline & & $\epsilon / \mathrm{kcal} \mathrm{mol}^{-1}$ & $\sigma / \AA$ & Charge \\
\hline Methanol $^{\mathrm{a}}$ & Oxygen & 0.170 & 3.07 & -0.700 \\
& Hydrogen & 0.000 & 0.00 & 0.435 \\
& Methyl group & 0.207 & 3.775 & 0.265 \\
DMSO $^{\mathrm{b}}$ & Sulfur & 0.23838 & 3.40 & 0.139 \\
& Oxygen & 0.07152 & 2.80 & -0.459 \\
& Methyl group & 0.29397 & 3.80 & 0.160 \\
\hline \hline
\end{tabular}

${ }^{\mathrm{a} O P L S}$ model (Refs. 27 and 28).

${ }^{\mathrm{b}}$ Reference 29.

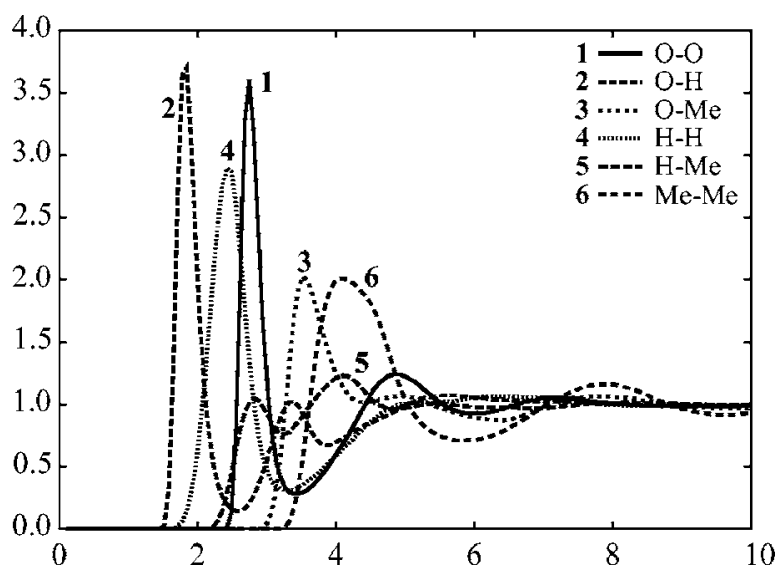

FIG. 2. Reference RDFs of methanol calculated by MD.

$$
\begin{aligned}
& n_{s}(\mathbf{r})=\left.\sum_{\alpha}^{N_{a}} w_{\alpha}(\mathbf{r}) n_{s}(\mathbf{r})\right|_{\alpha}, \\
& \sum_{\alpha}^{N_{a}} w_{\alpha}(\mathbf{r})=1 .
\end{aligned}
$$

The simple weight, $w_{\alpha}(\mathbf{r})=1 / N_{a}$, was used through our study. Because the quality of $\left\{\left.n_{s}(\mathbf{r})\right|_{\eta}\right\}$ far from the origin atom $\eta$ becomes worse, better weight function will improve results. This procedure is summarized in Scheme 1.

\section{COMPUTATIONAL DETAILS}

In the present method, any kind of functions can be used for $\left\{f_{i}^{\eta, s}\right\}$ in Eq. (2). In this article, we used roof functions for $\left\{f_{i}^{\eta, s}\right\}$,

$$
f_{i}^{\eta, s}(r)= \begin{cases}0 & \text { for } 0 \leqslant r \leqslant r_{i-1} \\ \frac{r-r_{i-1}}{r_{i}-r_{i-1}} & \text { for } r_{i-1} \leqslant r \leqslant r_{i} \\ \frac{r_{i+1}-r}{r_{i+1}-r_{i}} & \text { for } r_{i} \leqslant r \leqslant r_{i+1} \\ 0 & \text { for } r_{i+1} \leqslant r .\end{cases}
$$

The following function was used as the penalty function in Eq. (10):

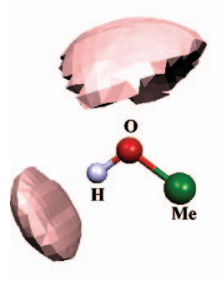

(a)

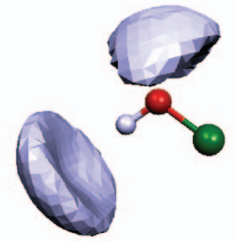

(b)

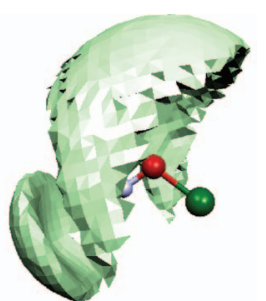

(c)
FIG. 3. (Color) Three dimensional SDF (MD) map of oxygen (a), of hydrogen (b), and of methyl group (c) in methanol. The isosurfaces of SDFs are drawn at $n(\mathbf{r})=3.0$. 


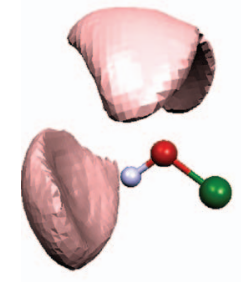

(a)

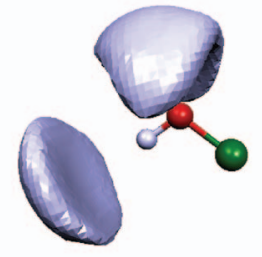

(b)

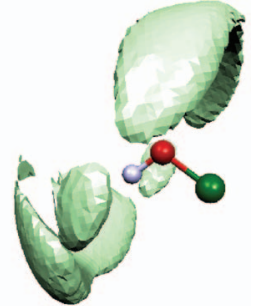

(c)
FIG. 4. (Color) Three dimensional RC-SDF map of oxygen (a), of hydrogen (b), and of methyl group (c) in methanol. The isosurfaces are the same as in Fig. 2.

$$
\phi(S)= \begin{cases}\alpha S^{4} & (S \leqslant 0) \\ 0 & (S \geqslant 0) .\end{cases}
$$

The $\alpha$ value can be determined arbitrary; in this work, we used $\alpha=15.0$. Although $n_{s}(\mathbf{r})$ becomes negative with this $\alpha$ in several regions, the negative value is small enough to neglect it (minimum value is -0.11 in this work).

In the calculation of the MD simulation, we use simple rigid potential model for methanol and DMSO with the intermolecular pair potential. All Lennard-Jones parameters and the fractional charges used for methanol and DMSO are listed in Table I.

MD simulations were carried out within the $N V T$ ensemble at $T=298.15 \mathrm{~K}$ under $1 \mathrm{~atm}$. In this simulation, the cubic periodic box was filled with 256 molecules. The simulation software used was MOLDY. ${ }^{18}$ Temperature was controlled with Nosé-Hoover thermostat. ${ }^{19,20}$

All of 3D figures are drawn with the help of MOLEKEL. ${ }^{21}$

\section{RESULTS AND DISCUSSION}

First, we performed MD calculations of methanol and DMSO to obtain their RDFs. Then, we evaluated RC-SDFs from the RDFs. For the purpose of comparison, we also calculated the SDF from the MD trajectory directly [SD$\mathrm{F}(\mathrm{MD})]$.

\section{A. Methanol}

The reference RDFs calculated directly by the MD method are shown in Fig. 2. The RDFs between oxygen and oxygen, oxygen and hydrogen, and hydrogen and hydrogen

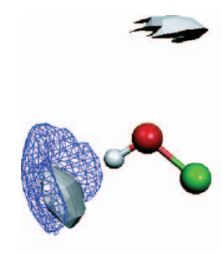

(a)

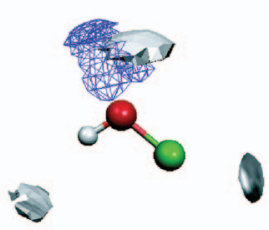

(b)

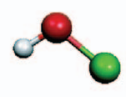

(c)
FIG. 5. (Color) Difference maps between RC-SDF and SDF(MD) of oxygen (a), of hydrogen (b), and of methyl group (c) in methanol. The region where the difference $\left[n_{s}(\mathbf{r})\right.$ of $\mathrm{RC}-\mathrm{SDF}-n_{s}(\mathbf{r})$ of $\left.\mathrm{SDF}(\mathrm{MD})\right]$ is larger than 5.0 is drawn with mesh and the region where the difference is less than -5.0 is drawn with solid surface.
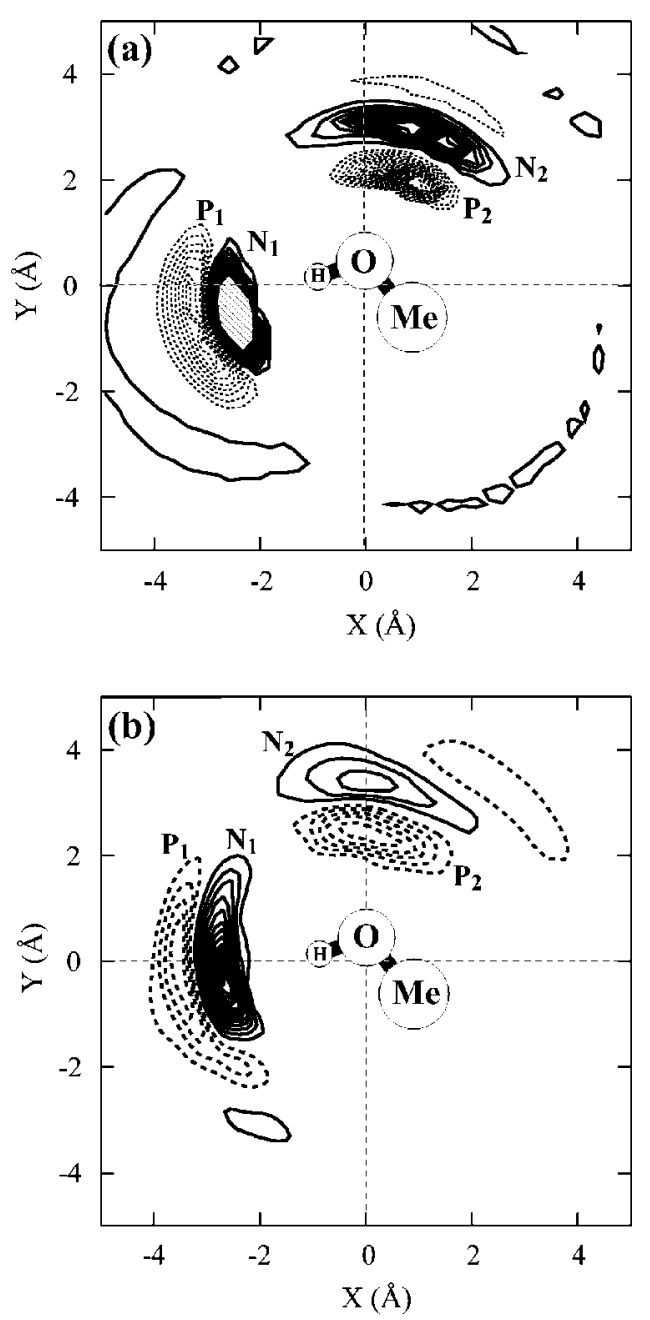

FIG. 6. Two dimensional map of charge density. (a) The contour of SD$\mathrm{F}(\mathrm{MD})$. The interval of contour is 0.010 for positive value and 0.015 for negative value. The region where negative value is less than -0.210 is shaded. (b) The contour of RC-SDF. The interval is the same as in (a).

display sharp peaks, while the RDFs between oxygen and methyl group, hydrogen and methyl group, and methyl group and methyl group exhibit broad peaks.

The SDF(MD) and the RC-SDF of oxygen site, hydrogen site, and methyl site are shown in Figs. 3 and 4, respectively. In SDF(MD), the distribution of oxygen (O) lies nearer to solute hydrogen site than the distribution of hydrogen $(\mathrm{H})$ and lies more separately from oxygen site than the distribution of hydrogen $(\mathrm{H})$. These distributions of SDF(MD) are well reproduced by RC-SDF, as shown in Fig. 4. The distribution of methyl group is mainly observed around oxygen and hydrogen sites but it is more delocalized. Both $\mathrm{SDF}(\mathrm{MD})$ and RC-SDF present essentially the same distribution of methyl group, while the distribution of RC-SDF is somewhat more localized around the solute oxygen site than that of SDF(MD) [compare Fig. 3(c) with Fig. 4(c)]. As shown by these examples, RC-SDF can produce well the distributions of SDF(MD) except for moderately delocalized distribution of methyl group.

To make the difference between RC-SDFs and SDF$\mathrm{s}(\mathrm{MD})$ clearer, the difference maps $\left[n_{s}(\mathbf{r})\right.$ of $\mathrm{RC}-\mathrm{SDF}-n_{s}(\mathbf{r})$ of $\mathrm{SDF}(\mathrm{MD})]$ are shown in Fig. 5. As mentioned above, 

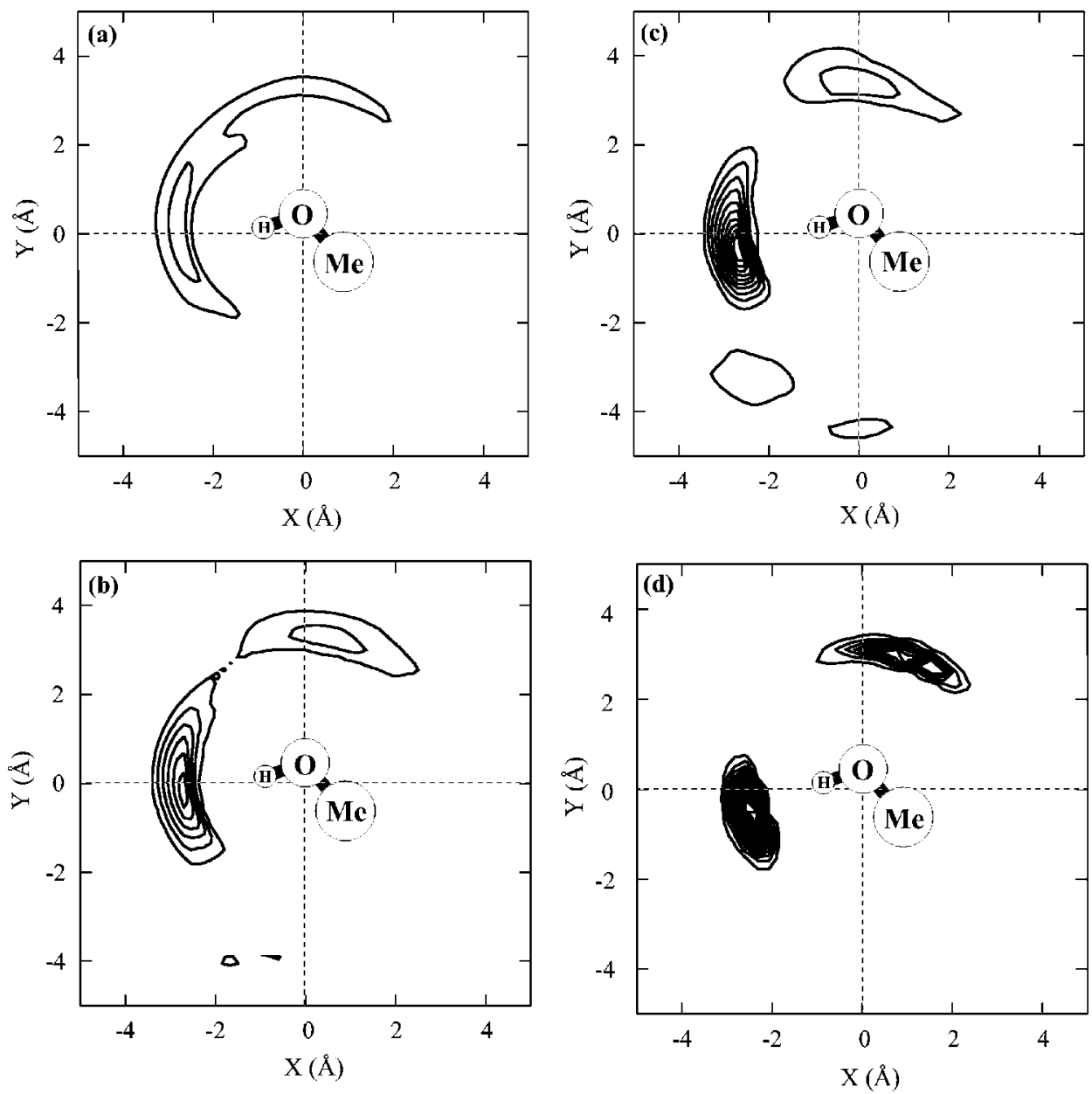

FIG. 7. Two dimensional RC-SDF map of oxygen in methanol with different angular momenta. The interval of contour value is 2 . (a) $n=2$, (b) $n$ $=6$, (c) $n=10$, and (d) the result obtained by MD.
RC-SDFs present almost same distribution with SDFs(MD).

To analyze the peak positions of these distributions, we show contour maps of charge density calculated by $n_{s}(\mathbf{r})$ of SDF(MD) and RC-SDF in Figs. 6(a) and 6(b). The charge density is represented by

$$
\rho_{q}(\mathbf{r})=\rho \sum_{\gamma}^{N_{a}} q_{\gamma} n_{\gamma}(\mathbf{r}),
$$

where $\rho$ is the number density of solvent and $q_{\gamma}$ is the charge of solvent $\gamma$ site. In the case of methanol, the negative distribution (solid line) mainly comes from the distribution (O) and the positive one (dashed line) comes from the distributions of hydrogen and methyl group. Because these distributions have little overlap between each other, the positive and negative regions can be recognized as the distributions of hydrogen/methyl group and oxygen group, respectively. By using this charge density maps, three different information

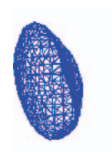

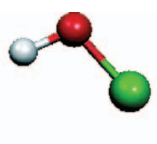

(a)
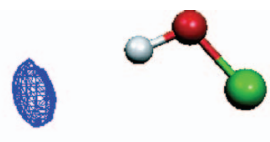

(b)
FIG. 8. (Color) Difference maps of RC-SDF (oxygen) between $n=2$ and $n=10$ (a) and $n=6$ and $n=10$ (b). The region where the difference is larger than 5.0 is shown. can be compiled to one figure. In Fig. 6(a), negative distribution is observed about $X=-2.34 \AA$ and $Y=-0.75 \AA\left(\mathrm{N}_{1}\right)$ and positive distribution $\left(\mathrm{P}_{1}\right)$ is outside this negative area. Another positive distribution is observed about $X=0.69 \AA$ and $Y=2.08 \AA\left(\mathrm{P}_{2}\right)$ and negative one $\left(\mathrm{N}_{2}\right)$ is outside of this negative area. The considerably localized distributions $\mathrm{N}_{1}$ and $\mathrm{P}_{2}$ and their very high peaks indicate that oxygen and hydrogen atoms of solvent form strong bonding interaction with hydrogen and oxygen of solute, respectively. On the other hand, the distributions $\mathrm{N}_{2}$ and $\mathrm{P}_{1}$ are very broad. In the case of RC-SDF, the distributions $\mathrm{N}_{1}$ and $\mathrm{P}_{2}$ are more localized than the distributions $\mathrm{P}_{1}$ and $\mathrm{N}_{2}$, as observed in the case of SDF(MD) [see Figs. 6(a) and 6(b)]. Although the peaks of these distributions are smaller than those of $\mathrm{SDF}(\mathrm{MD})$, the peaks of RC-SDF are at almost the same position as those of $\mathrm{SDF}(\mathrm{MD})$.

To investigate how much the RC-SDFs depend on the order of real solid harmonics used in Eq. (3), we calculated the distributions of solvent oxygen with real solid harmonics up to orders $n=2, n=6$, and $n=10$, as shown in Figs. 7(a)-7(c), respectively. ${ }^{22}$ In Fig. 7(d), SDF(MD) of oxygen is also shown. In Fig. 7(a), broad distribution is observed around the solute. As $n$ increases, this broad distribution separates into two distributions [Fig. 7(b)] concomitantly with the increase of the height and the distribution approaches the SDF(MD). The difference maps of RC-SDFs between $n=2$ and $n=10$ and between $n=6$ and $n=10$ are 


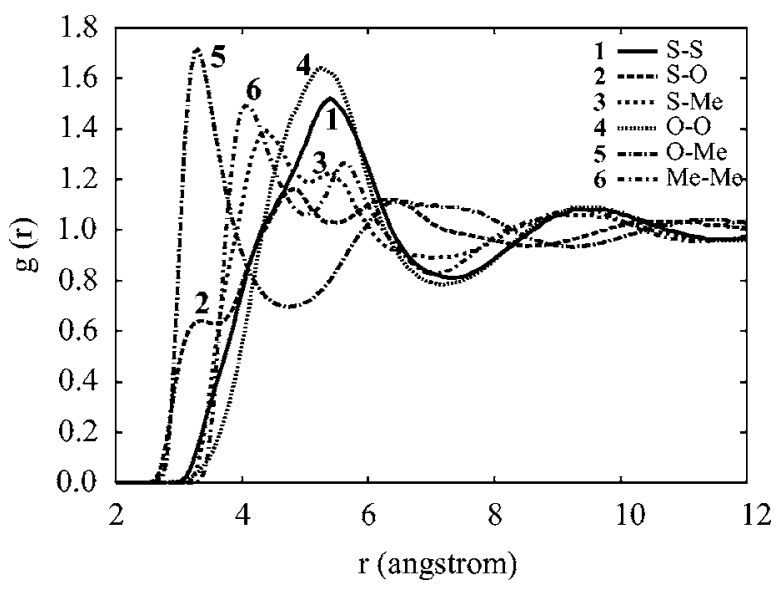

FIG. 9. Reference RDFs of DMSO calculated by the MD method.

shown on Fig. 8. Although the difference in shape of RCSDF between $n=2$ and RC-SDF $n=6$ is considerably large [Fig. 8(a)], the difference between $n=6$ and $n=10$ becomes very small, as shown in Fig. 8(b). This result indicates that the increase in the order $n$ improves the shape of SDF.

The computational time of these calculations is very short ${ }^{23}$ for instance, the calculation of RC-SDF with real solid harmonics $(n=10)$ takes about $5 \mathrm{~min}$ for the distribution of oxygen, about $8 \mathrm{~min}$ for that of hydrogen, and about 4 min for that of methyl group. Although the basis functions and grid size are different from those employed in our previous work, ${ }^{15}$ the computational time considerably decreases by using roof functions and discrete real solid harmonics. ${ }^{24}$

\section{B. DMSO}

In the case of DMSO, several broad peaks are observed in the reference RDFs (Fig. 9), unlike methanol in which the reference RDFs exhibit sharp peaks attributed to hydrogen bonding. These RDFs clearly indicate that the solvation structure becomes more complexed than that of methanol.

The SDFs(MD) and RC-SDFs are shown in Figs. 10 and 11. The solvation structure by SDFs(MD) (Fig. 10) is similar to that of DMSO-water mixture reported by Vishnyakov et $a l^{4}{ }^{4} \mathrm{SDF}(\mathrm{MD})$ of sulfur displays broad distribution near the solute methyl group $\left(\mathrm{D}_{1}\right)$ and very broad distribution distant from the solute $\left(\mathrm{D}_{2}\right)$ [see Fig. 10(a)]. These distributions can be reproduced well by RC-SDF, as shown in Fig. 11(a). SDF(MD) of oxygen, on the other hand, presents rela-

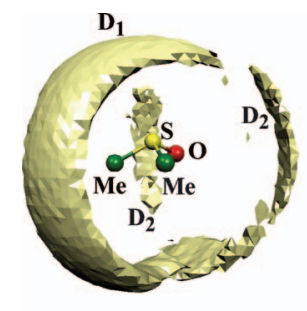

(a)

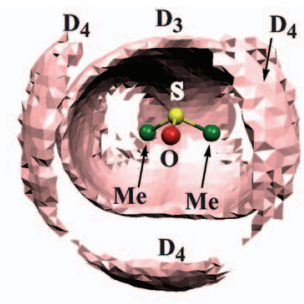

(b)

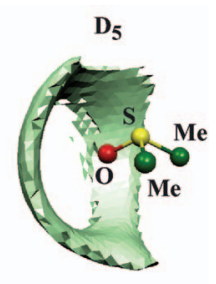

(c)
FIG. 10. (Color) Three dimensional SDF(MD) map of sulfur (a), of oxygen (b), and of methyl group (c). The isosurfaces of SDFs are drawn at $n(\mathbf{r})$ $=2.0$ for $(\mathrm{a})$ and $(\mathrm{b})$, and 2.9 for $(\mathrm{c})$.

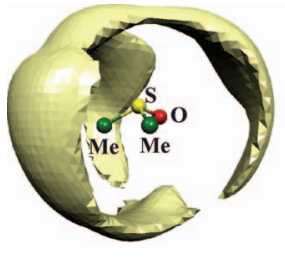

(a)

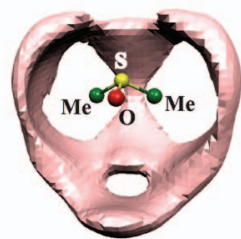

(b)

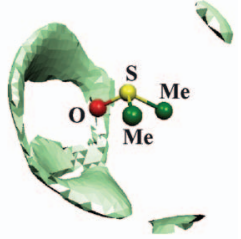

(c)
FIG. 11. (Color) Three dimensional RC-SDF(MD) map of sulfur (a), of oxygen (b), and of methyl group (c). The isosurfaces are the same as in Fig. 8.

tively localized distribution around solute methyl group $\left(\mathrm{D}_{3}\right)$ and broad distribution $\left(\mathrm{D}_{4}\right)$ distant from solute oxygen site. The relatively localized distribution is attributed to moderately strong electrostatic interaction between oxygen and methyl group. RC-SDF can reproduce well this localized distribution $\left(D_{3}\right)$. However, the broad distribution $\left(D_{4}\right)$ of oxygen is moderately different between $\mathrm{SDF}(\mathrm{MD})$ and RC-SDF. The broad distribution of solvent methyl group is presented around solute by SDF(MD), as shown in Fig. 10(c). RC-SDF can present well this broad one, as shown in Fig. 11(c).

The difference maps $\left[n_{s}(\mathbf{r})\right.$ of RC-SDF- $n_{s}(\mathbf{r})$ of SD$\mathrm{F}(\mathrm{MD})]$ are shown in Fig. 12. Although broad distributions cannot be reproduced by RC-SDF, the important distributions, such as the one between solute oxygen and solute methyl groups, are reproduced very well, as mentioned above.

\section{CONCLUSIONS}

In our previous work, ${ }^{15}$ we proposed a new method to calculate RC-SDF from RDFs. In this work, we successfully refined the method by using roof functions and discrete real solid harmonics. Here, RC-SDF is computed under the three conditions described below. By integrating $\left.n_{s}(\mathbf{r})\right|_{\eta}$ around the solute site, the RDF must be reproduced (condition I). The sets of $\left\{\left.n_{s}(\mathbf{r})\right|_{\eta}\right\}$ at each solute site must be consistent with each other in 3D space around solute (condition II). $\left.n_{s}(\mathbf{r})\right|_{\eta}$ must be positive in 3D space (condition III). Although the equation to be solved is not linear because of condition III, RC-SDF can be obtained iteratively with rea-

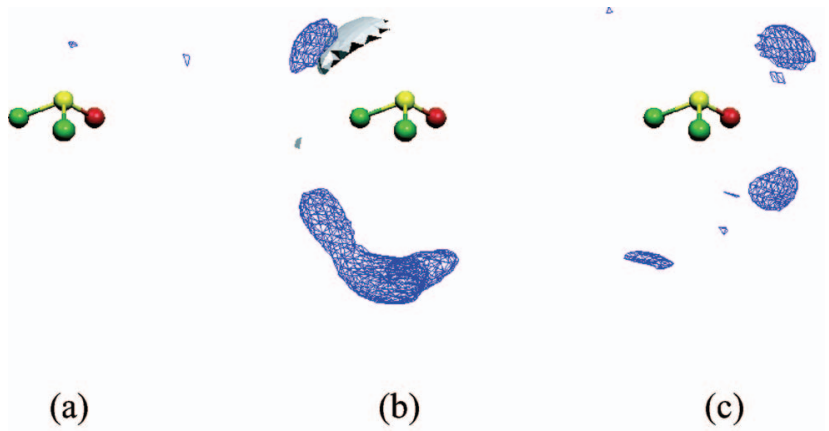

FIG. 12. (Color) Difference maps between RC-SDF and SDF(MD) of sulfur (a), of oxygen (b), and of methyl group (c) in DMSO. The region where the difference $\left[n_{s}(\mathbf{r})\right.$ of RC-SDF $-n_{s}(\mathbf{r})$ of $\left.\mathrm{SDF}(\mathrm{MD})\right]$ is larger than 2.5 is drawn with mesh and the region where the difference is less than -2.5 is drawn with solid surface. 
sonable computational time. The obtained RC-SDF can not only produce well-defined 3D solvation structure of methanol but also diffuse one of DMSO.

This method can present RC-SDF from any kind of RDFs with reasonable computational cost. In other words, this method can be combined with methods which present RDFs, such as neutron scattering and RISM self-consistent field (SCF). ${ }^{25,26}$ Combination of these methods provides much clearer understanding of solvation event than the usual RDFs.

\section{ACKNOWLEDGMENTS}

This work has been financially supported in part by the Grant-in Aid for Scientific Research on Priority Areas "Water and biomolecules" (430-16041223 and 430-18031019), by "Control of Dynamic Complexes" (420), by the Grant-in Aid for Encouragement of Young Scientists (17750012), and by the Grand-in Aid for General Research (15350012). One of the authors (D.Y.) thanks the Grant-in Aid for JSPS Fellows. All of the authors were supported by the Ministry of Education, Science, Sports, and Culture (MONBU SHO) of Japan. Some computations were carried out with computer system of IMS (Okazaki).

${ }^{1}$ J. R. Asher, N. L. Doltsinis, and M. Kaupp, J. Am. Chem. Soc. 126, 9854 (2004)

${ }^{2}$ I. M. Svishchev and P. G. Kusalik, J. Chem. Phys. 99, 3049 (1993).

${ }^{3}$ I. M. Svishchev and A. Yu. Zassetsky, J. Chem. Phys. 113, 7432 (2000).

${ }^{4}$ A. Vishnyakov, A. P. Lyubartsev, and A. Laaksonen, J. Phys. Chem. A 105, $1702(2001)$

${ }^{5}$ J. P. Hansen and I. R. McDonald, Theory of Simple Liquids, 2nd ed. (Academic, London, 1986).

${ }^{6}$ P. H. Fries and G. N. Patey, J. Chem. Phys. 82, 429 (1985).

${ }^{7}$ M. Lombardero, C. Martín, S. Jorge, F. Lado, and E. Lomba, J. Chem. Phys. 110, 1148 (1999)

${ }^{8}$ M. Ikeguchi and J. Doi, J. Chem. Phys. 103, 5011 (1995).

${ }^{9}$ D. Beglov and B. Roux, J. Phys. Chem. B 101, 7821 (1997).

${ }^{10}$ V. Shapovalov, T. N. Truong, A. Kovalenko, and F. Hirata, Chem. Phys. Lett. 320, 186 (2000).

${ }^{11}$ A. K. Soper, Physica B 213-214, 448 (1995).
${ }^{12}$ A. K. Soper, J. Chem. Phys. 101, 6888 (1994).

${ }^{13}$ A. K. Soper, C. Andreani, and M. Nardone, Phys. Rev. E 47, 2598 (1993).

${ }^{14}$ H. Sato and F. Hirata, Bull. Chem. Soc. Jpn. 74, 1831 (2001).

${ }^{15}$ D. Yokogawa, H. Sato, and S. Sakaki, J. Chem. Phys. 123, 211102 (2005).

${ }^{16}$ K. Takahashi, X. Peng, K. Komine, M. Ohdaira, Y. Abe, T. Sugimura, K. Goto, H. Fuchigami, M. Yamada, and K. Watanabe, Proceedings of the Seventh International Conference on High Performance Computing and Grid in Asia Pacific Region, 2004 (unpublished).

${ }^{17}$ Unfortunately, there is a typo in Ref. 15 . The correct element of the matrix $\mathbf{B}$ [Eq. 7(b)] is

$$
\begin{aligned}
B_{(s)}^{\alpha, i, m}= & \sum_{i}^{N_{p}} f_{i}^{\alpha, s}\left(\left|\mathbf{r}-\mathbf{Q}_{\alpha}\right|\right) S_{l, m}(\theta, \phi) \sum_{\gamma}\left(N_{a} \delta_{\alpha, \gamma}-1\right) \\
& \times\left(\sum_{i}^{N} f_{i}^{\gamma, s}\left(\left|\mathbf{r}-\mathbf{Q}_{\gamma}\right|\right) C_{0,0}^{\gamma, s, i}+f_{f}^{\gamma, s}\left(\left|\mathbf{r}-\mathbf{Q}_{\gamma}\right|\right)\right) .
\end{aligned}
$$

In this work, $\Sigma f_{i}^{\gamma, s}\left(\left|\mathbf{r}-\mathbf{Q}_{\gamma}\right|\right) C_{0,0}^{\gamma, s, i}+f_{f}^{\gamma, s}\left(\left|\mathbf{r}-\mathbf{Q}_{\gamma}\right|\right)$ in the previous work is represented as the function $f^{\gamma, s}\left(\left|\mathbf{r}-\mathbf{Q}_{\gamma}\right|\right)$.

${ }^{18}$ K. Refson, Comput. Phys. Commun. 126, 310 (2000)

${ }^{19}$ S. Nosé, J. Chem. Phys. 81, 511 (1984).

${ }^{20}$ W. G. Hoover, Phys. Rev. A 31, 1695 (1985).

${ }^{21}$ P. Flükiger, H. P. Lüthi, S. Portmann, and J. Weber, Molekel 4.0, Swiss Center for Scientific Computing, Manno, Switzerland, 2000.

${ }^{22}$ The total numbers of real solid harmonics are $8(n=2), 48(n=6)$, and $120(n=10)$.

${ }^{23}$ All of the calculations were performed with a single CPU (Pentium IV $\mathrm{EE})$ at $3.20 \mathrm{GHz}$. In the calculation of DMSO, our program requires about 120 Mbytes of memory.

${ }^{24}$ In this work, the numbers of the coefficients of expansion are 1560, 1920, and 2040 for RC-SDF of oxygen, hydrogen, and methyl group, respectively. The number of grid points is $60^{3}$ for all of the RC-SDFs. In the previous work, the number of the coefficients of expansion is 1080 for RC-SDF of oxygen and the number of grid points is $50^{3}$. The calculation of water is required about $1 \mathrm{~h}$ with the same machine.

${ }^{25}$ S. Ten-no, F. Hirata, and S. Kato, J. Chem. Phys. 100, 7443 (1994).

${ }^{26}$ H. Sato, F. Hirata, and S. Kato, J. Chem. Phys. 105, 1546 (1996).

${ }^{27}$ W. L. Jorgensen, J. Phys. Chem. 90, 1276 (1986).

${ }^{28}$ W. L. Jorgensen, J. D. Madura, and C. J. Swenson, J. Am. Chem. Soc. 106, 6638 (1984).

${ }^{29}$ A. Luzar and D. Chandler, J. Chem. Phys. 98, 8160 (1993).

${ }^{30}$ R. Thomas, C. B. Shoemaker, and K. Eriks, Acta Crystallogr. 21, 12 (1966). 